\title{
Duration of fecal shedding of Shiga toxin- producing Escherichia coli among children with hemolytic uremic syndrome
}

Lucas I. Lucarelli, M.D. ${ }^{a}$, Laura F. Alconcher, M.D. ${ }^{a}$, Verónica Arias, Biochemist ${ }^{a}$ and Jimena Galavotti, Biochemist ${ }^{a}$

\begin{abstract}
Introduction. Knowing the duration of fecal shedding of Shiga toxin-producing Escherichia coli (STEC) among patients with hemolytic uremic syndrome would be useful to control disease transmission.

Objectives. 1) To analyze the characteristics of STEC shedding duration. 2) To assess the association with sex, age, need of dialysis, antibiotics, and STEC serotypes.

Population and methods. Prospective, observational, longitudinal, and analytical study in the 2013-2019 period. Stool cultures were done upon admission and every 5-7 days until 2 negative results were obtained. Shedding duration was defined as the period from diarrhea onset to the first negative result. STEC was confirmed with polymerase chain reaction detection of stx1, stx2, and $r f b O 157$ genes. The mean $(95 \% \mathrm{CI})$ and percentile values of the STEC shedding duration were estimated, and the studied outcome measures were compared using the $t$ test.

Results. A total of 43 patients were included. The mean duration of shedding was 10.2 days (95 \% CI: 8.92-11.59), range: 3-22 days. After 15 days, $90 \%$ of patients had a negative stool culture. There were no differences in terms of sex $(p=0.419)$, age $(p=0.937)$, need of dialysis $(p=0.917)$, antibiotics $(p=0.147)$ or serotype $(p=0.231)$

Conclusion. Fifteen days after the onset of diarrhea, $90 \%$ of patients had a negative stool culture, and all patients had one after 22 days. No association was observed between the duration of shedding and studied outcome measures. Key words: enterohemorrhagic Escherichia coli, bacterial shedding, transmission period, stools.
\end{abstract}

a. Hospital Interzonal Dr. José Penna,

Bahía Blanca, Argentina.

E-mail address:

Lucas Lucarelli, M.D.:

1_lucarelli@hotmail.com

http: / / dx.doi.org/ 10.5546/ aap.2021.eng.39

To cite: Lucarelli LI, Alconcher LF, Arias V, Galavotti J. Duration of fecal shedding of Shiga toxin-producing Escherichia coli among children with hemolytic uremic syndrome. Arch Argent Pediatr 2021;119(1):39-43.
Funding:

None.

Conflict of interest:

None.

Received: 7-27-2020

Accepted: 9-7-2020

\section{INTRODUCTION}

The diagnosis of hemolytic uremic syndrome (HUS) is based on the diagnostic triad of microangiopathic hemolytic anemia, thrombocytopenia, and varying degrees of acute kidney involvement. In Argentina, HUS is endemic; the median number of cases reported annually for the 20142018 period was 314 and the median number of cases among children younger than 5 years was $245 .{ }^{1}$ Most cases are associated with diarrhea and, in $65 \%$, Shiga toxin-producing Escherichia coli (STEC) infection is confirmed. ${ }^{2}$ HUS may be transmitted by eating STEC contaminated food, contact with the feces of carrier animals or contact with the feces of a sick person (diarrhea and/or HUS) and/or their close contacts.

Studies about diarrhea outbreaks in daycare centers and kindergartens in different places around the world have demonstrated that the shedding of E. coli O157 may be prolonged and intermittent. ${ }^{3-8}$ Knowing the duration of fecal shedding of STEC among patients with HUS would be useful to safely reintroduce these children into society and thus control secondary HUS transmission.

\section{OBJECTIVES}

1. To analyze the characteristics of STEC shedding duration among patients with HUS.

2. To assess the association between shedding duration and the following outcome measures: sex, age, need of dialysis, antibiotic use, and STEC serotypes. 


\section{MATERIALS AND METHODS}

This was a prospective, observational, longitudinal, and analytical study. The results of stool cultures from children with HUS seen in the period between January 2013 and December 2019 at the Sentinel Unit of Hospital Interzonal General (HIG) Dr. José Penna, in Bahía Blanca, Argentina, were analyzed. Stool samples were collected upon hospital admission and every 5-7 days until 2 consecutive negative results were obtained in order to detect intermittent shedding cases. STEC infection was confirmed with polymerase chain reaction (PCR) detection of stx1, st $x 2$, and rfbO157 genes. ${ }^{9}$

Patients who did not have a stool culture and those with a negative result were excluded. Patients who did not meet the number and/or deadline for sample collection were left out. To consider the possibility of intermittent shedding, the duration of fecal shedding of STEC was defined as the period (in days) from the onset of diarrhea to the first of 2 consecutive negative stool cultures obtained 48 hours apart.

To calculate the sample size necessary to estimate the duration of shedding, 12 patients were selected to be part of a pilot sample, with a mean duration of 19.08 days and a 13day deviation. For a $95 \%$ confidence and an absolute error of 4 days (relative error of $20 \%$ ), the necessary sample size was estimated at 41 patients. The Epidat 3.1 software was used.

Mean values and their $95 \%$ confidence intervals (CIs) and percentiles were estimated. A cumulative frequency histogram was developed. The $t$ test for mean differences was done to assess the relation between the duration of shedding and the outcome measures of sex, age (younger or older than 2 years), need for dialysis

FIGURE 1. Cumulative frequency histogram of the duration of shedding of Shiga toxin-producing Escherichia coli among patients with hemolytic uremic syndrome

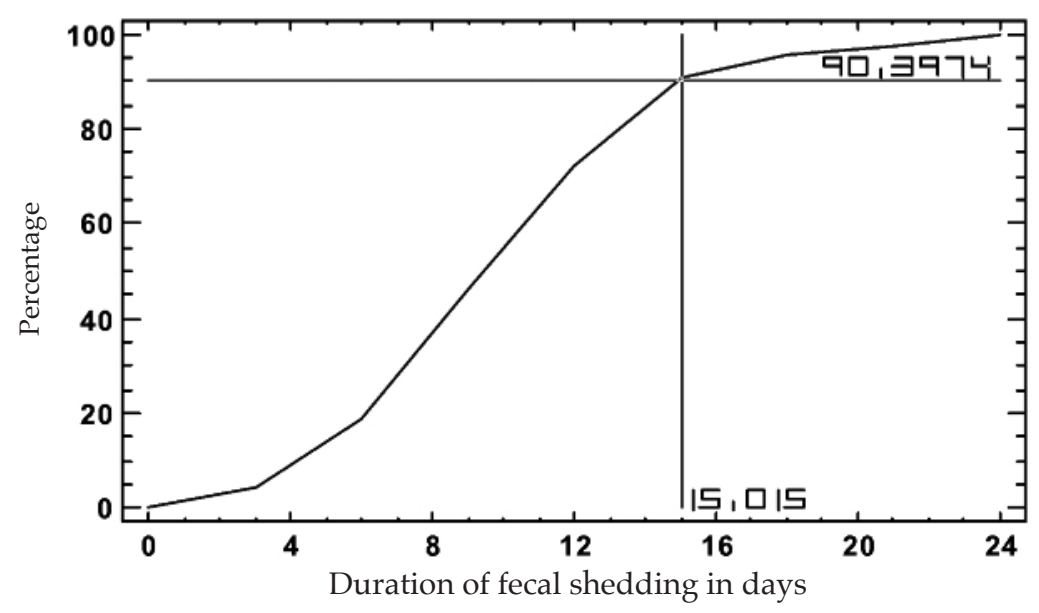

FIGURE 2. Histogram of duration of shedding

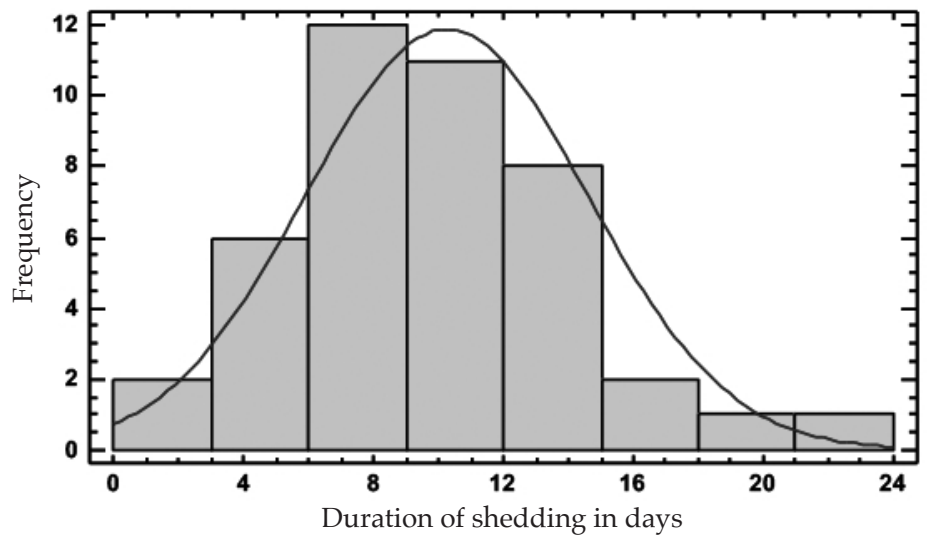

The duration of shedding shows a normal distribution. A Gaussian-like behavior is observed. 
(yes/no), antibiotic use (yes/no), and STEC serotypes (O157/ non-O157). A value of $p<0.05$ was considered significant. The Statgraphics Centurion XVI and PSSS v22 software programs were used. The study was approved by both the Ethics and Research Committees of HIG Dr. J. Penna. Written informed consents were obtained.

\section{RESULTS}

During the 2013-2019 period, 88 patients with HUS were seen. Of them, 37 were excluded: 36 because their stool culture was negative and 1 because no stool culture was done. Out of the 51 patients with a positive stool culture for STEC, 8 were left out because they did not meet the number and/or deadline for consecutive stool sample collection.

A total of 43 patients were included; $22(51 \%)$ were girls and $24(56 \%)$ were younger than 2 years. Twenty-three patients $(53 \%)$ required dialysis. Four patients $(9 \%)$ received antibiotic therapy before admission. E. coli $\mathrm{O} 157$ was isolated in 29 subjects $(67 \%)$.

The mean duration of fecal shedding was 10.2 days (95\% CI: 8.25-11.39 days). In $90 \%$ of patients, the stool culture was negative after 15 days, and in all, after 22 days (Figure 1). The outcome measure duration of shedding showed a normal curve distribution (Figure 2 and Table 1).

In only 2 cases, intermittent fecal shedding was detected, with a total duration of 12 and 21 days, respectively. No statistically significant association was observed between the duration of fecal shedding of STEC and age, sex, need of dialysis, antibiotic use or isolated serotype (Table 2).

TABLE 1. Percentiles for the duration of fecal shedding of Shiga toxin-producing Escherichia coli among patients with hemolytic uremic syndrome $(n=43)$

\begin{tabular}{lc}
\hline Percentile & Days of shedding \\
\hline $1 \%$ & 3 \\
$5 \%$ & 4 \\
$10 \%$ & 5 \\
$25 \%$ & 7 \\
$50 \%$ & 10 \\
$75 \%$ & 13 \\
$90 \%$ & 15 \\
$95 \%$ & 17 \\
$99 \%$ & 22 \\
\hline
\end{tabular}

\section{DISCUSSION}

As reported in different epidemiological studies, person-to-person STEC transmission plays a major role in the endemic behavior of HUS in Argentina. Such transmission is especially related to kindergartens, daycare centers, and close family contacts. ${ }^{5,10,11}$ It has been observed recently that one-third of patients with HUS had positive STEC family contacts who were mostly asymptomatic, but were a potential risk for transmission. ${ }^{10}$ Interpersonal transmission occurs very easily because the infective dose is very low (100-500 microorganisms). ${ }^{11}$

Belongia et al. (1993) did not find evidence of person-to-person STEC transmission in daycare centers when children with diarrhea were reintroduced after 2 negative stool cultures. ${ }^{7}$ The same criterion is used in our country for the reintroduction of HUS patients to daycare centers and kindergartens, but STEC detection in stools by PCR is an expensive, complex test that is not easily available at all hospitals. For this reason, knowing the duration of fecal shedding of STEC may be a useful tool to prevent new cases. In our study, stool culture negativization was observed in $90 \%$ of patients with HUS 15 days after diarrhea onset, and in all patients, after 22 days.

Most information about the duration of fecal shedding of STEC was obtained from patients with diarrhea corresponding to the $\mathrm{O} 157$ serotype. Shash et al. (1996) studied an outbreak at a daycare center and reported a mean duration of shedding of E. coli O157 of 29 days (range: 11-57 days). ${ }^{4}$ Miliwebsky et al. (2007) studied 4 diarrhea events in daycare centers in Argentina

TABLE 2. Relation between the duration of shedding of Shiga toxin-producing Escherichia coli among children with hemolytic uremic syndromeand selected clinical, demographic, and microbiological outcome measures $(n=43)$

\begin{tabular}{lcccc}
\hline Factor & Category & N (days) & Mean & $p$ value \\
\hline Sex & Male & 21 & 10.81 & 0.419 \\
Age (years) & Female & 22 & 9.73 & \\
& $<2$ & 24 & 10.21 & 0.937 \\
STEC serotypes & Non-O157 & 14 & 10.36 & 0.917 \\
& O157 & 29 & 10.21 & \\
Antibiotics & Yes & 4 & 7.25 & 0.147 \\
\multirow{4}{*}{ Need of dialysis } & No & 39 & 10.56 & \\
& Yes & 23 & 11.00 & 0.231 \\
& No & 20 & 9.40 & \\
\hline
\end{tabular}

STEC: Shiga toxin-producing Escherichia coli. 
that affected children, staff, and family members. The isolated serotype was non-O157 in 3 of them. Shedding was prolonged and similar between the $\mathrm{O} 157$ and non-O157 serotypes (with a maximum of 37 and 31 days for the O26:H11 serotype and 19 days for the O145:NM serotype). ${ }^{5}$

Few data have been reported in patients with HUS. Karch et al. (1995) studied 53 patients with diarrhea caused by STEC and 25 patients with HUS, and found that the mean duration of fecal shedding was significantly longer among patients with HUS (13 versus 21 days, respectively, $p<0.001) .{ }^{8}$ On the contrary, in the diarrhea and HUS outbreak caused by enteroaggregative E. coli O104:H4 in 2011 in Germany, the duration of fecal shedding of STEC lasted a median of 1314 days among patients with HUS versus 3334 days among patients with diarrhea. ${ }^{12} \mathrm{~A}$ longer shedding duration may be due to an infective dose of more than 106 colony forming units (CFU)/g. ${ }^{8}$ In 2015, Matussek reported an average duration of fecal shedding of STEC of 23 days in 7 patients with HUS and 20 days in patients with diarrhea. ${ }^{13}$

Antibiotic therapy in patients with diarrhea due to STEC may increase the risk for HUS; therefore, it is not recommended. ${ }^{14}$ However, in the German outbreak, the group of patients with severe HUS was treated with eculizumab and also prophylactic azithromycin to reduce the risk for meningitis, which resulted in a significantly shorter shedding duration of STEC. Only 1 patient out of 22 treated with azithromycin $(4.5 \%)$ had a shedding duration of more than 28 days versus 35 out of $43(81 \%)$ of those who did not receive antibiotics. ${ }^{15}$ Only 4 patients in our series had received antibiotics before admission. Although the duration of shedding was shorter in these patients, it was not statistically significant. To date, there are no conclusive data about the usefulness of antibiotics to shorten STEC shedding duration among patients with HUS.

Although a longer STEC shedding has been reported among younger patients, both in the setting of diarrhea outbreaks and among patients with HUS, such statistical association was not observed in our study.,12 Although data about the duration of shedding of STEC have already been published, most studies are focused on patients with diarrhea and some isolated HUS cases, except for the German group, which studied a different $E$. coli category.

Fifteen days after the onset of diarrhea, $90 \%$ of patients had a negative stool culture. By resolution of the Ministry of Health, patients with HUS should not be reintroduced to daycare centers and/or kindergartens until they have at least 2 consecutive negative stool cultures. If this is not possible, and considering the potential severity of a STEC infection and the probability of developing HUS, it seems cautious to establish a 22-day period as the safety limit to rejoin social activities. Knowing this information allows to establish when a patient should be reintroduced to society at a low risk for transmission. We believe that this information would be especially useful in Argentina to prevent new cases due to the endemic behavior of the disease and the major role played by interpersonal transmission.

\section{CONCLUSION}

Fifteen days after the onset of diarrhea, $90 \%$ of patients had a negative stool culture, and all patients had one after 22 days. No statistically significant association was observed between the duration of fecal shedding of STEC and age, sex, need of dialysis, antibiotic use or isolated serotype. This information may be useful to prevent the onset of secondary STEC infections in settings where culture negativization cannot be established by PCR.

\section{Acknowledgments}

We would like to thank María Eugenia Elorza, $\mathrm{PhD}$ in Economics, and Fernanda Villarreal, $\mathrm{PhD}$ in Economics, for the statistical analysis done at Universidad Nacional del Sur.

\section{REFERENCES}

1. Argentina. Ministerio de Salud. Vigilancia de Síndrome Urémico Hemolítico. Boletín Integrado de Vigilancia. 2019;477(SE 50):47. [Accessed on: June 11 ${ }^{\text {th }}, 2020$ ]. Available at: https: / / www.argentina.gob.ar/sites / default / files/201912-biv_477.pdf.

2. Rivas M. Epidemiología del Síndrome Urémico Hemolítico en Argentina. Situación actual e innovaciones diagnósticas. 2016. [Accessed on: June $11^{\text {th }}, 2020$ ]. Availableat:http:/ / www.sap.org.ar/uploads/archivos / / files_dra-rivas-epidemiologia-del-sindrome-uremicohemolitico-en-argentina-situacion-actual-e-innovacionesdiagnosticas_1494446234.pdf.

3. Swerdlow DL, Griffin PM. Duration of faecal shedding of Escherichia coli O157:H7 among children in day-care centres. Lancet. 1997; 349(9054):745-6.

4. Shash S, Hoffman R, Shillam P, Wilson B. Prolonged fecal shedding of Escherichia coli O157:H7 during an outbreak at day care center. Clin Infect Dis. 1996; 23(4):835-6.

5. Miliwebsky E, Deza N, Chinen I, Martínez Espinosa E, et al. Prolonged fecal shedding of Shiga toxin-producing Escherichia coli among children attending day-care centers in Argentina. Rev Argent Microbiol. 2007; 39(2):90-2.

6. Raffaelli RM, Paladini M, Hanson H, Kornstein L, et al. Child care-associated outbreak of Escherichia coli O157:H7 
and hemolytic uremic syndrome. Pediatr Infect Dis J. 2007; 26(10):951-3.

7. Belongia EA, Osterholm MT, Soler JT, Ammend DA, et al. Transmission of Escherichia coli 0157:H7 Infection in Minnesota Child Day-care Facilities. JAMA. 1993; 269(7):883-8.

8. Karch H, Rüssman H, Schmidt H, Schwarzkopf A, et al. Long-term shedding and clonal turnover of enterohemorrhagic Escherichia coli O157:H7 in diarrheal diseases. J Clin Microbiol. 1995; 33(6):1602-65.

9. Servicio de Fisiopatogenia, Departamento de Bacteriología. Manual de Procedimientos. Diagnóstico y Caracterización de Escherichiacoli productor de toxina Shiga O157 y no-O157 a partir de especímenes clínicos. Buenos Aires: INEI-ANLIS “Carlos G. Malbrán"; 2011.

10. Alconcher LF, Rivas M, Lucarelli LI, Galavotti J, et al. Shiga toxin-producing Escherichia Coli in household members of children with hemolytic uremic syndrome. Eur J Clin Microbiol Infect Dis. 2020; 39(3):427-32.

11. Zotta CM, Chinen I, Lavayén S, Cepeda M, et al. Portación de Escherichia coli en convivientes de casos de síndrome urémico hemolítico. Salud(i)Ciencia. 2015; 21(2):136-41.

12. Vonberg R, Höhle M, Aepfelbacher M, Bange FC, et al. Duration of fecal shedding of Shiga toxin-producing Escherichia coli O104:H4 in patients infected during the 2011 outbreak in Germany: a multicenter study. Clin Infect Dis. 2013; 56(8):1132-40.

13. Matussek A, Einemo I, Jogenfors A, Löfdahl S, et al. Shiga toxin-producing Escherichia coli in diarrheal stool of Swedish children: evaluation of polymerase chain reaction screening and duration of Shiga toxin shedding. J Pediatric Infect Dis Soc. 2016; 5(2):147-51.

14. Wong CS, Jelacic S, Habeeb RL, Watkins SL, et al. The risk of the hemolytic-uremic syndrome after antibiotic treatment of Escherichia coli O157:H7 infections. N Engl J Med. 2000; 342(26):1930-6.

15. Nitschke M, Sayk F, Hartel C, Härtel C, et al. Association between azithromycin therapy and duration of bacterial shedding among patients with Shiga toxin-producing enteroaggregative Escherichia coli O104:H4. JAMA. 2012; 307(10):1046-52. 